\title{
On Efficient and Low-Complexity Decoding of Binary LDPC-Coded CSK Signals for GNSS Links with Increased Data Rates
}

\author{
Rémi Chauvat \\ TELECOM Laboratory \\ École Nationale de l'Aviation Civile \\ Toulouse, France \\ remi.chauvat@ recherche.enac.fr
}

\author{
Axel Garcia-Pena \\ TELECOM Laboratory \\ École Nationale de l'Aviation Civile \\ Toulouse, France \\ garcia-pena@ recherche.enac.fr
}

\author{
Matteo Paonni \\ Joint Research Centre \\ European Commission \\ Ispra, Italy \\ matteo.paonni@ec.europa.eu
}

\begin{abstract}
GNSS with high data rate links are of interest to accommodate new needs and applications (e.g. precise positioning, authentication, reduction of TTFFD). In this context, a binary LDPC-coded CSK signal is an attractive candidate to increase data rates with a high data recovery robustness. However, such a proposal requires an increase of receiver's computational complexity with respect to receivers for current coded DSSS/BPSK GNSS links. The computational complexity required for data recovery is analysed in this article and insights on crucial technical choices are given for the reception of binary LDPC-coded CSK signals. CSK demodulation is shown to dominate the overall computational cost and the use of digital chip-matched filtering prior to demodulation is proposed to reduce this cost. In addition, iterative demapping, which is crucial to optimize the power efficiency of binary LDPC-coded CSK links is also shown to have high computational complexity. Therefore, low-complexity iterative demapping strategies are studied and simple yet efficient solutions are proposed.
\end{abstract}

\section{INTRODUCTION}

Global navigation satellite systems (GNSS) with high data rate links could be envisioned in the future due to the emergence of new needs and applications. In particular, the deployment of precise positioning generally requires the exploitation of a large amount of data. Indeed, precise positioning may necessitate the transmission of a significant quantity of data corrections (e.g. for clock, ephemeris, ionosphere...). Another major new need for GNSS is authentication which is becoming a highly demanded service for current and future GNSS signals. Authentication also requires additional information to be transmitted such as the cryptographic signatures or keys. Moreover, GNSS signals with higher data rates than current ones could provide a reduction of the time-to-firstfix data (TTFFD) through an increase of the rate at which the transmission of clock error corrections and ephemeris data is repeated.

Nevertheless, the increase of the GNSS signal data rate is not trivial since GNSS links use direct-sequence spreadspectrum (DSSS) signals in order to perform accurate positioning. Historically, the data component of the GNSS signal used DSSS with a binary phase shift-keying (BPSK) data modulation. Therefore, considering DSSS/BPSK signals, a symbol rate increase would necessitate either an increase of the chip rate, further broadening the occupied bandwidth, or a reduction of the number of chips within pseudo-random noise (PRN) sequences, reducing the inter-system and intra-system orthogonality properties of signals. None of these two previous options is desirable. Therefore, the best option may consist in implementing higher order data modulations.

A high order data modulation specifically designed to increase data rates in DSSS systems is the cyclic code-shift keying (CSK) modulation [1], a particular form of orthogonal modulation. The high energy efficiency of CSK makes the solution especially attractive with respect to other high order modulations such as $M$-ary amplitude and phase modulations (for $M>4$ ). 256-ary CSK signals are already used in the L6 link of the Quasi-Zenith Satellite System (QZSS) [2], for centimeter-level augmentation.

In addition, recent GNSS signals make use of channel codes to enhance their link robustness. Classical CCSDS convolutional codes have been widely used in Galileo and GPS, amongst others. However, recent practices favour modern channel codes (e.g. low-density parity-check (LDPC) codes in GPS L1C subframes 2 and 3, BeiDou B1C and B2a signals) which are able to further improve the power efficiency of links [3], [4].

The use of binary LDPC-coded CSK signals has been shown to be an attractive solution in order to increase data rates in GNSS with high data recovery robustness [5], [6]. Unfortunately, while the CSK modulation allows a low-complexity implementation at transmitter side, requiring only a single shift register for the generation of CSK symbols, the reception of CSK signals has a computational complexity which is increased with respect to DSSS/BPSK links. However, though CSK acquisition complexity has been subject to previous works as in [7], to the best of our knowledge, CSK demodulation strategies and their computational cost have been only partly and scarcely discussed, with some results and proposals mainly disseminated in [5], [8], [9]. Also, while low complexity iterative LDPC decoding has been extensively studied in the literature, efficient low-complexity iterative 
demapping for the CSK modulation, or more generally for orthogonal modulations [10], [11] has been less discussed.

It is one of the purpose of this paper to provide an accurate characterization of the computational complexity required for the data recovery of coded CSK and DSSS/BPSK signals. The data recovery structures are described in a general framework. Various decoder configurations are presented with their error rate performance and computational cost. Simple yet efficient low-complexity decoder implementations are exposed.

After this introduction, generic structures for GNSS data transmission and recovery are presented in sections II and III. Many receiver configurations are introduced with their error rate performance in section IV. After that, a computational complexity analysis of receivers is conducted in section $\mathrm{V}$. A complexity reduction strategy for iterative CSK demapping is proposed in section VI. Finally, section VII concludes the article.

\section{TRANSMITTER STRUCTURE}

\section{A. Generic structure}

The simplified structure of a generic transmitter of GNSS data component is depicted in Fig. 1. First, a channel encoder generates a codeword $\mathbf{x}$ from the information sequence $\mathbf{m}$. Second, an optional interleaver (П) performs a permutation on the codeword bits. For simplicity, we assume here that the interleaver length is equal to the codeword length. Finally, a spreading modulator maps the codeword to a sequence of symbols. After that, up-conversion at the carrier frequency $f_{c}$ and power amplification are performed before the resulting signal $s(t)$ is radiated from the transmit antenna.

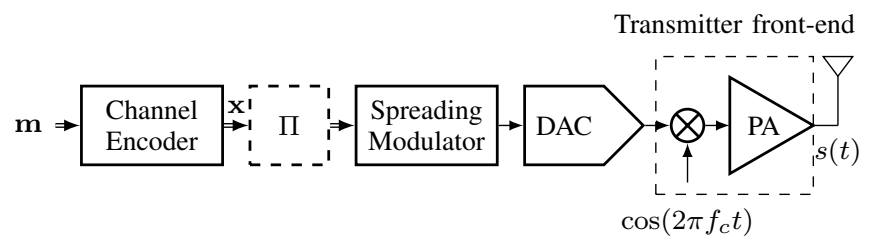

Fig. 1. Simplified generic transmitter of GNSS data component

In GNSS, channel encoding typically uses convolutional codes but most recent links tend to favour LDPC codes [4], able to improve the power efficiency of the data link. The spreading modulations considered are generally DSSS/BPSK, or more recently DSSS with binary offset carrier (BOC). Note that the data demodulation strategies for BPSK and BOC are similar.

\section{B. DSSS/BPSK and CSK data modulations}

The structure of DSSS/BPSK signals limits the achievable data rates to a single bit per PRN sequence duration. Denoting $L$ the number of chips of PRN sequences used, $\mathbf{c}_{\mathrm{f}} \triangleq$ $\left[c_{0}^{\mathrm{f}}, c_{1}^{\mathrm{f}}, \ldots, c_{L-1}^{\mathrm{f}}\right]$, the fundamental (bipolar, i.e. $c_{l}^{\mathrm{f}} \in\{ \pm 1\}$ ) PRN sequence and assuming a transmission of one new BPSK symbol $d_{q} \in\{ \pm 1\}$ at every epoch $q$ corresponding to the start of a new PRN sequence, the transmitted DSSS/BPSK signal is

$$
\begin{aligned}
s(t) & \triangleq A \sum_{q=-\infty}^{+\infty} \sum_{l=0}^{L-1} \gamma_{l, q} p\left(t-[l+q L] T_{c}\right) \cos \left(2 \pi f_{c} t\right) \\
& \triangleq \tilde{s}(t) \cos \left(2 \pi f_{c} t\right)
\end{aligned}
$$

where $\gamma_{l, q}=d_{q} c_{l}^{\mathrm{f}}, A$ denotes the transmitter front-end amplification factor, and $p(t)$ is the rectangular chip waveform of chip period duration $T_{c}$, typically used in GNSS.

A $M$-ary CSK modulation has the potential to increase the data rate by $U \triangleq \log _{2}(M)$ with respect to the DSSS/BPSK case. $M$-ary CSK is a quasi-orthogonal modulation whose symbol set is composed of $M$ cyclicallyshifted versions of $\mathbf{c}_{\mathrm{f}}$. Bits mapped by the $\mu^{\text {th }}$ symbol are denoted $\mathbf{b}_{\mu} \triangleq\left[b_{0}^{\mu}, b_{1}^{\mu}, \ldots, b_{U-1}^{\mu}\right]$. The $\mu^{\text {th }}$ CSK symbol corresponds to the $\mu^{\text {th }}$ cyclically-shifted version of $\mathbf{c}_{\mathrm{f}}$ denoted as $\left.\mathbf{c}_{\mu} \triangleq\left[c_{0}^{\mu}, c_{1}^{\mu}, \ldots, c_{L-1}^{\mu}\right]\right)$. Using the notation $c_{\mathrm{f}}[i] \equiv c_{i}^{\mathrm{f}}, c_{l}^{\mu}$ is expressed $c_{l}^{\mu}=c_{\mathrm{f}}[(l+\mu) \bmod L]$. Note that $\mathbf{c}_{0} \equiv \mathbf{c}_{\mathrm{f}}$ corresponds to the fundamental PRN code. Considering equiprobable and independent data bits and denoting the transmitted CSK symbol at epoch $q$ as $\overline{\mathbf{c}}_{q} \triangleq\left[\bar{c}_{0, q}, \bar{c}_{1, q}, \ldots, \bar{c}_{L-1, q}\right] \in$ $\left\{\mathbf{c}_{\mu}\right\}_{0 \leq \mu<M}$, the transmitted GNSS CSK signal can also be modeled with (1) but using $\gamma_{l, q}=\bar{c}_{l, q}$.

\section{Binary LDPC channel coding}

In the sequel, we will generally assume the use of binary LDPC channel codes, aiming at further enhancing the power efficiency of the data component.

A binary LDPC code $\mathcal{C}$ is a linear block code [4] defined by the null-space of a sparse $m \times n$ parity-check matrix $\mathbf{H}$ having coefficients $h_{i, j}(0 \leq i<m, 0 \leq j<n)$ over the Galois field $\mathcal{G F}(2)$. $\mathbf{H}$ can be equivalently represented by a bipartite (Tanner) graph [12]. On this Tanner graph, each row of $\mathbf{H}$ is represented by a check node $(\mathrm{CN})$ while each column is represented by a variable node ( $\mathrm{VN})$. The $j^{\text {th }} \mathrm{VN}$ is connected to a $i^{\text {th }} \mathrm{CN}$ by an edge iff $h_{i, j} \neq 0$.

\section{RECEIVER STRUCTURE}

\section{A. Assumptions}

GNSS receivers frequently use a heterodyne receiver architecture with sampling at intermediate frequency (IF) [13], [14]. For the complexity analysis, such a structure will be assumed in the sequel. Note however that the analysis would remain similar in a baseband sampling context, the differences being the removal of a need for digital carrier wipe-off and different typical reception parameters such as the sampling frequency.

In addition, we will consider that perfect synchronization is achieved (e.g. typically using an orthogonal pilot component for acquisition and tracking). Also, for simplicity, we will assume the sampling frequency $f_{s}$ to be an integer multiple of the chip frequency ${ }^{1}$, i.e. $f_{s}=\alpha f_{c}$ with $\alpha \in \mathbb{N}^{*}$ and $f_{c}=1 / T_{c}$.

\footnotetext{
${ }^{1}$ Note however that, from a positioning accuracy point of view, this case should be avoided in practice [15].
} 


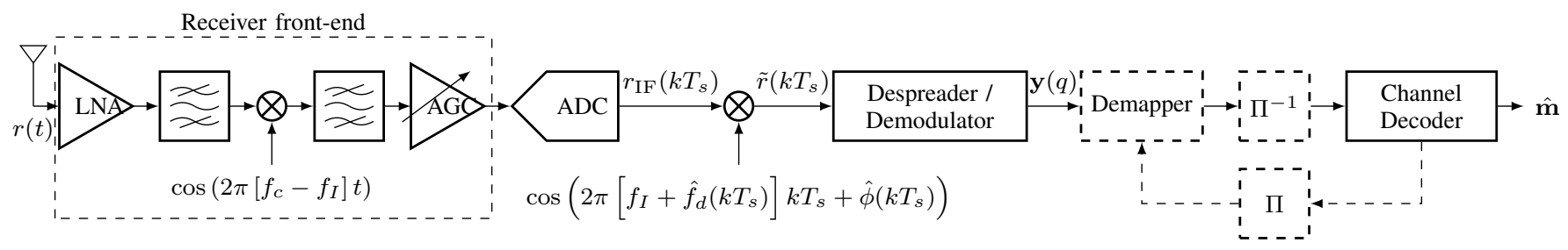

Fig. 2. Simplified generic data recovery structure with sampling at IF for GNSS data component (dashed blocks are specific to BICM/BICM-ID)

\section{B. Generic structure}

A typical receiver structure with sampling at IF is depicted in Fig. 2, the simplified front-end structure being given only as example. A carrier wipe-off follows the analog-to-digital conversion (ADC). Then, because of the use of a binary error correcting code, data recovery structures can generally be separated in three distinct tasks. First, demodulation is performed which provides the symbols' likelihoods. Second, for modern binary channel codes that use bit likelihood metrics, a step denoted here as demapping ${ }^{2}$ computes these bit likelihoods from the demodulator's output. Finally, after desinterleaving, channel decoding is performed. This is the traditional structure of the bit interleaved coded modulation (BICM) reception [16]. Note that BICM with iterative demapping (BICM-ID) is also possible, illustrated in Fig. 2 by the feedback link between the channel decoder and the demapper.

\section{Received signal model after sampling at IF}

We consider the received signal model after an ideal frontend processing and analog-to-digital conversion. Considering a Doppler shift $f_{d}(t)$, a timing delay $\tau(t)$, a residual phase $\phi(t)$ and an additive noise $n_{\mathrm{IF}}(t)$, the sampled received data component at intermediate frequency can be expressed

$$
\begin{aligned}
r_{\mathrm{IF}}\left(k T_{s}\right)= & n_{\mathrm{IF}}\left(k T_{s}\right)+A^{\prime} \tilde{s}\left(k T_{s}-\tau\left(k T_{s}\right)\right) \\
& \times \cos \left(2 \pi\left[f_{I}+f_{d}\left(k T_{s}\right)\right] k T_{s}+\phi\left(k T_{s}\right)\right)
\end{aligned}
$$

where $T_{s}=1 / f_{s}, A^{\prime}$ denotes the receiver front-end amplification factor and $f_{I}$ is the receiver IF. After carrier wipeoff with perfect Doppler shift and residual phase estimation $\left(\forall k: \hat{f}_{d}\left(k T_{s}\right)=f_{d}\left(k T_{s}\right), \hat{\phi}\left(k T_{s}\right)=\phi\left(k T_{s}\right)\right)$, the resulting signal becomes

$$
\begin{aligned}
\tilde{r}\left(k T_{s}\right)= & r_{\mathrm{IF}}\left(k T_{s}\right) \cos \left(2 \pi\left[f_{I}+\hat{f}_{d}\left(k T_{s}\right)\right] k T_{s}+\hat{\phi}\left(k T_{s}\right)\right) \\
\triangleq & \tilde{n}\left(k T_{s}\right)+\frac{A^{\prime}}{2} \tilde{s}\left(k T_{s}-\tau\left(k T_{s}\right)\right) \\
& \times\left\{1+\cos \left(4 \pi\left[f_{I}+f_{d}\left(k T_{s}\right)\right] k T_{s}+2 \phi\left(k T_{s}\right)\right)\right\}
\end{aligned}
$$

where $\tilde{n}\left(k T_{s}\right)$ denotes the noise component after carrier wipeoff. Note that the high frequency component of (5) will be later removed by the despreading process.

\footnotetext{
${ }^{2}$ Note that this is a slight redefinition from common usage in communications literature.
}

\section{Coherent demodulation}

Perfect code delay estimation is assumed $\left(\forall k: \hat{\tau}\left(k T_{s}\right)=\right.$ $\tau\left(k T_{s}\right)$ ). For mathematical convenience, we also assume that this delay is a constant multiple of the sampling period, i.e. $\forall k: \hat{\tau}\left(k T_{s}\right)=v T_{s}, v \in \mathbb{N}$. Omitting the sampling period $T_{s}$, coherent DSSS/BPSK demodulation necessitates a single correlator whose output can be written

$$
y(q)=\sum_{k=\alpha q L+v}^{\alpha(q+1) L-1+v} \tilde{r}(k) c_{\mathrm{f}, \alpha}([k-v] \bmod \alpha L)
$$

where $\mathbf{c}_{\mathrm{f}, \alpha}$ is the sampled fundamental PRN sequence, i.e. $\mathbf{c}_{\mathrm{f}, \alpha}=\left[c_{\mathrm{f}, \alpha}(0), c_{\mathrm{f}, \alpha}(1), \ldots, c_{\mathrm{f}, \alpha}(\alpha L-1)\right] \triangleq \mathbf{c}_{\mathrm{f}} \otimes \mathbf{1}_{\alpha}, \otimes$ denoting the Kronecker product and $\mathbf{1}_{\alpha}$ being the all-one row vector with length $\alpha$. However, as for other forms of orthogonal modulations, CSK demodulation is achieved using a bank of correlators, each matched to a particular CSK symbol (cf. Fig. 3).

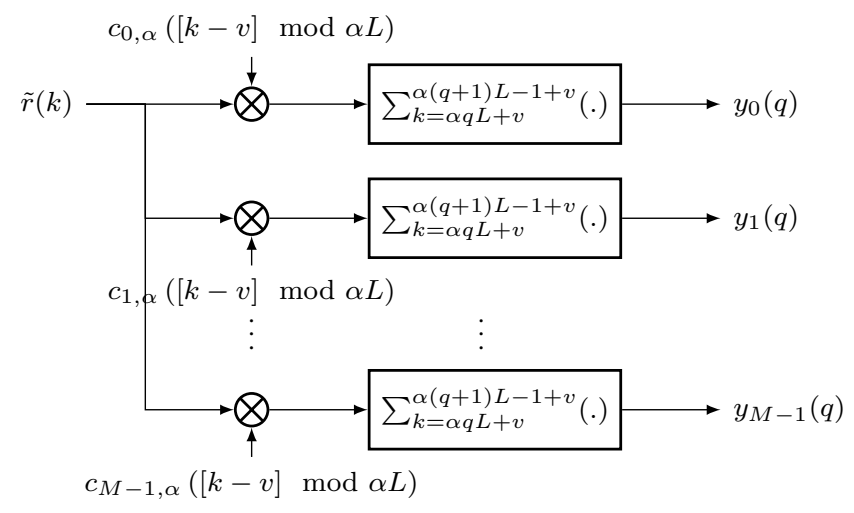

Fig. 3. Correlator-bank based CSK demodulator

The $\mu^{\text {th }}$ correlator output is expressed similarly to (6) with $\mathbf{c}_{\mu, \alpha}$ instead of $\mathbf{c}_{\mathrm{f}, \alpha}$, i.e.

$$
y_{\mu}(q)=\sum_{k=\alpha q L+v}^{\alpha(q+1) L-1+v} \tilde{r}(k) c_{\mu, \alpha}([k-v] \bmod \alpha L)
$$

In the sequel, $\mathbf{y}(q)$ will denote the demodulator output (column) vector for the $q^{\text {th }}$ symbol, i.e. $\mathbf{y}(q) \triangleq$ $\left[y_{0}(q), y_{1}(q), \ldots, y_{M-1}(q)\right]^{T}$.

Furthermore, because of the circularly-shifted symbols of CSK, demodulation can also be performed in the Fourier domain. Denoting $\tilde{\mathbf{r}}_{q} \triangleq[\tilde{r}(\alpha q L+v), \tilde{r}(\alpha q L+v+1), \ldots, \tilde{r}(\alpha([q+$ $1] L)+v-1)]$ the digital sequence corresponding to the $q^{\text {th }}$ 
transmitted symbol, the Fourier-domain CSK demodulation can be expressed

$$
\mathbf{y}(q)=\operatorname{IDFT}\left\{\operatorname{DFT}\left\{\tilde{\mathbf{r}}_{q}\right\} \circ \operatorname{DFT}\left\{\mathbf{c}_{\mathbf{f}, \alpha}\right\}^{*}\right\}
$$

where $\circ$ denotes the Hadamard product, $\operatorname{IDFT}\{$.$\} and DFT \{$. are the inverse and forward discrete Fourier transforms, respectively. Fourier-domain CSK demodulation is depicted in Fig. 4. Note that both $\tilde{\mathbf{r}}_{q}$ and $\mathbf{c}_{0, \alpha}$ can be zero padded in

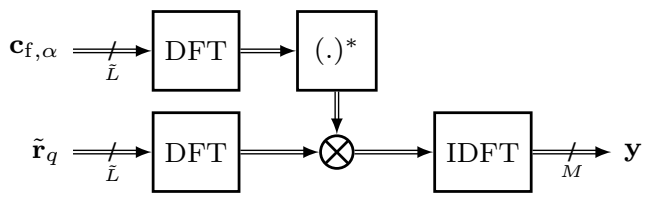

Fig. 4. Fourier-domain CSK demodulator

order to have power-of-two lengths $\tilde{L} \triangleq 2^{\left\lceil\log _{2}(\alpha L)\right\rceil}$, therefore allowing efficient implementation using fast Fourier transform (FFT)/IFFT algorithms.

\section{E. Coherent demapping}

When a binary LDPC error correcting code is used, the computation of log-likelihood ratios (LLR) of a posteriori probabilities (APP) of bits is required. The extrinsic LLR obtained from this generic APP LLR expresses as

$$
\begin{aligned}
L_{\mathrm{e}}\left(b_{j}(q) \mid \mathbf{y}(q)\right) & =\ln \frac{p\left(b_{j}(q)=0 \mid \mathbf{y}(q)\right)}{p\left(b_{j}(q)=1 \mid \mathbf{y}(q)\right)}-\ln \frac{p\left(b_{j}(q)=0\right)}{p\left(b_{j}(q)=1\right)} \\
& \triangleq L\left(b_{j}(q) \mid \mathbf{y}(q)\right)-L\left(b_{j}(q)\right)
\end{aligned}
$$

where $b_{j}(q)$ is the $j^{\text {th }}$ bit of the received sequence corresponding to the $q^{\text {th }}$ transmitted symbol. Note from (9) that the APP LLR is $L\left(b_{j}(q) \mid \mathbf{y}(q)\right)$ while a priori bit LLR is $L\left(b_{j}(q)\right)$. For the sequel, we will omit the index $q$, for clarity.

1) Coherent DSSS/BPSK demapping: Considering DSSS/BPSK signals in AWGN channel, the APP LLR metric simply writes

$$
L\left(b_{j} \mid y\right)=\frac{2}{\sigma_{n}^{2}} y
$$

where $\sigma_{n}^{2}$ is the variance of noise samples.

2) Coherent CSK demapping: Considering $M$-ary CSK signals in AWGN channel, after some algebraic manipulations, the APP LLR metric can be written

$$
L\left(b_{j} \mid \mathbf{y}\right)=\ln \frac{\sum_{\mu: b_{j}^{\mu}=0} \exp \left(\frac{y_{\mu}}{\sigma_{n}^{2}}-\mathbf{b}_{\mu} \mathbf{L}_{\mathrm{a}}^{T}\right)}{\sum_{\mu^{\prime}: b_{j}^{\mu^{\prime}}=1} \exp \left(\frac{y_{\mu^{\prime}}}{\sigma_{n}^{2}}-\mathbf{b}_{\mu^{\prime}} \mathbf{L}_{\mathrm{a}}^{T}\right)}
$$

where $\mathbf{L}_{\mathrm{a}} \triangleq\left[L\left(b_{0}\right), L\left(b_{1}\right), \ldots, L\left(b_{U-1}\right)\right]$ is the vector of a priori LLR for the CSK symbol's bits. This a priori information can be provided by the channel decoder and is used in BICMID. The resulting iterative demapper structure is depicted in Fig. 5.

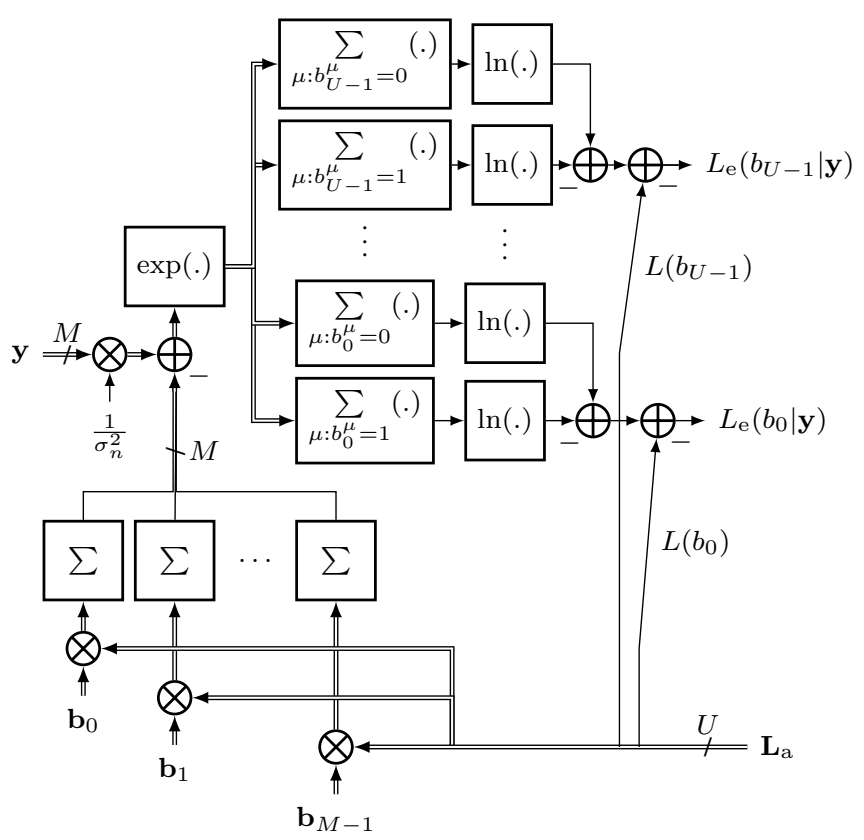

Fig. 5. Architecture for iterative log MAP CSK demapping

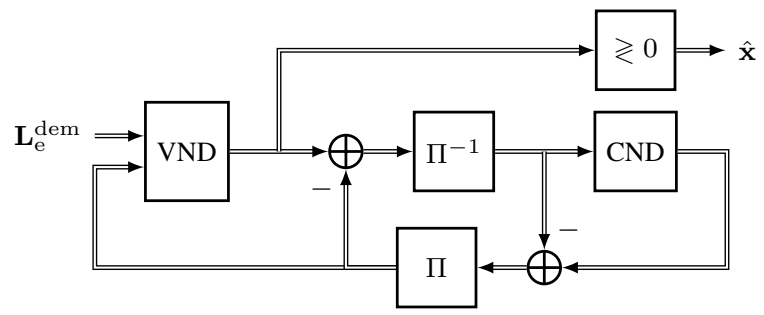

Fig. 6. Equivalent representation of an LDPC decoder

Assuming equi-probable bits and no feedback of information from the outer decoder, the extrinsic LLR becomes

$$
L_{\mathrm{e}}\left(b_{j} \mid \mathbf{y}\right)=\ln \frac{\sum_{\mu: b_{j}^{\mu}=0} \exp \left(\frac{y_{\mu}}{\sigma_{n}^{2}}\right)}{\sum_{\mu^{\prime}: b_{j}^{\mu^{\prime}}=1} \exp \left(\frac{y_{\mu^{\prime}}}{\sigma_{n}^{2}}\right)}
$$

and is used in (non-iterative) BICM demapping.

\section{F. Binary LDPC decoding}

In practice, LDPC decoding is performed iteratively [4]. The classical (log-domain) sum-product algorithm (SPA) is considered here. For simplicity and ease of use with an iterative demapper, we consider the standard flooding schedule [4]. Assuming non-iterative BICM demapping, the structure of the LDPC decoder is depicted in Figure 6. In the sequel, $\mathbf{L}_{\mathrm{e}}^{\mathrm{dem}} \triangleq\left[L_{\mathrm{e}}^{\mathrm{dem}}[0], L_{\mathrm{e}}^{\mathrm{dem}}[1], \ldots, L_{\mathrm{e}}^{\mathrm{dem}}[n-1]\right]$ will denote the (desinterleaved) demapper's output corresponding to the codeword $\mathrm{x}$. At every decoding iteration, a variable node decoder (VND) combines check messages computed at the check node decoder (CND) and messages from the (desinterleaved) demapper's output.

Denoting $I_{d e c}$ the maximum number of LDPC decoding iterations and $\mathcal{N}(i)($ resp. $\overline{\mathcal{N}}(j))$ the set of neighbouring nodes 
of the VN $i$ (resp. $\mathrm{CN} j$ ) such that $0 \leq i<n$ and $0 \leq j<$ $m$, the check message from $\mathrm{CN} j$ to $\mathrm{VN} i$ at iteration $l+1$ $\left(0 \leq l<I_{d e c}\right)$ can be written

$$
\bar{M}_{j \rightarrow i}^{(l+1)}=2 \tanh ^{-1}\left[\prod_{i^{\prime} \in \overline{\mathcal{N}}(j) \backslash i} \tanh \left(\frac{M_{i^{\prime} \rightarrow j}^{(l)}}{2}\right)\right]
$$

with $M_{i \rightarrow j}^{(l)}$ the message passed from $\mathrm{VN} i$ to $\mathrm{CN} j$ at iteration $l$, which expresses

$$
M_{i \rightarrow j}^{(l)}= \begin{cases}L_{\mathrm{e}}^{\mathrm{dem}}[i]+\sum_{j^{\prime} \in \mathcal{N}(i) \backslash j} \bar{M}_{j \rightarrow i}^{(l)} & l \neq 0 \\ L_{\mathrm{e}}^{\operatorname{dem}}[i] & l=0\end{cases}
$$

At every iteration $l+1$, the APP LLR for the codeword bit $i$ is computed with

$$
L_{\mathrm{APP}}^{(l+1)}[i]=L_{\mathrm{e}}^{\mathrm{dem}}[i]+\sum_{j^{\prime} \in \mathcal{N}(i)} \bar{M}_{j \rightarrow i}^{(l)}
$$

and the codeword estimate $\hat{\mathbf{x}}$ is computed from the sign of (16). A codeword check generally follows which computes the syndrome $\hat{\mathbf{x}} \mathbf{H}^{T}$ and finishes the decoding procedure if the syndrome is an all-zero vector.

\section{CSK RECEIVER CONFIGURATIONS}

Demodulation, demapping and binary LDPC decoding techniques presented in section III will be considered as reference configurations in this article. More precisely, the demapping algorithm use the bitwise maximum a posteriori (MAP) rule. Concerning the binary LDPC decoding, the SPA is the optimal iterative decoding algorithm in the sense that it is bitwise MAP-optimal for cycle-less codes. However, for both demapping and LDPC decoding, there also exist suboptimal variants that may produce sufficient performance at a lower computational complexity. Some sub-optimal strategies are presented hereafter.

\section{A. Sub-optimal coherent demapping algorithms}

Equations (12) and (13) can be expressed recursively in terms of the Jacobian logarithm $\ln (\exp (a)+\exp (b))=$ $\max (a, b)+\ln (1+\exp (-|a-b|)) \triangleq \max ^{\star}(a, b)$ such that

$$
\begin{aligned}
& L\left(b_{j} \mid \mathbf{y}\right)=\max ^{\star}\left(\frac{y_{\mu_{0}}}{\sigma_{n}^{2}}-\mathbf{b}_{\mu_{0}} \mathbf{L}_{\mathrm{a}}^{T}, \max ^{\star}\left(\frac{y_{\mu_{1}}}{\sigma_{n}^{2}}-\mathbf{b}_{\mu_{1}} \mathbf{L}_{\mathrm{a}}^{T}, \ldots\right)\right) \\
& \left.-\max ^{\star}\left(\frac{y_{\mu_{\frac{M}{2}}}}{\sigma_{n}^{2}}-\mathbf{b}_{\mu_{\frac{M}{2}}} \mathbf{L}_{\mathrm{a}}^{T}, \max ^{\star}\left(\frac{y_{\mu_{\frac{M}{2}+1}}}{\sigma_{n}^{2}}-\mathbf{b}_{\mu_{\frac{M}{2}+1}} \mathbf{L}_{\mathrm{a}}^{T}, \ldots\right)\right)\right)
\end{aligned}
$$

in BICM-ID where $\mu_{i}(0 \leq i<M)$ denotes symbols' indices which depend on $j$, and

$$
\begin{aligned}
L_{\mathrm{e}}\left(b_{j} \mid \mathbf{y}\right) & =\max \star\left(\frac{y_{\mu_{0}}}{\sigma_{n}^{2}}, \max \star\left(\frac{y_{\mu_{1}}}{\sigma_{n}^{2}}, \ldots\right)\right) \\
& -\max \star\left(\frac{y_{\mu_{M / 2}}}{\sigma_{n}^{2}}, \max \star\left(\frac{y_{\mu_{M / 2+1}}}{\sigma_{n}^{2}}, \ldots\right)\right)
\end{aligned}
$$

in BICM. Note that this formulation is interesting since $0<$ $\ln (1+\exp (-|a-b|)) \leq \ln (2)$, thus easily allowing the storage in look-up table (LUT) of the only elements of (17) and (18) requiring computationally complex operations.

A simplification of (17) and (18), denoted as max log MAP, occurs when approximating $\max ^{\star}(a, b)$ by $\max (a, b)$, leading to

$L\left(b_{j} \mid \mathbf{y}\right) \approx \max _{\mu: b_{j}^{\mu}=0}\left(\frac{y_{\mu}}{\sigma_{n}^{2}}-\mathbf{b}_{\mu} \mathbf{L}_{\mathrm{a}}^{T}\right)-\max _{\mu^{\prime}: b_{j}^{\mu^{\prime}}=1}\left(\frac{y_{\mu^{\prime}}}{\sigma_{n}^{2}}-\mathbf{b}_{\mu^{\prime}} \mathbf{L}_{\mathrm{a}}^{T}\right)$

for BICM-ID and

$$
L_{\mathrm{e}}\left(b_{j} \mid \mathbf{y}\right) \approx \frac{1}{\sigma_{n}^{2}}\left[\max _{\mu: b_{j}^{\mu}=0}\left(y_{\mu}\right)-\max _{\mu^{\prime}: b_{j}^{\mu^{\prime}}=1}\left(y_{\mu^{\prime}}\right)\right]
$$

for BICM, respectively.

\section{B. Sub-optimal LDPC decoding algorithms}

Using $\alpha_{i \rightarrow j} \triangleq \operatorname{sign}\left(M_{i \rightarrow j}\right)$ and $\beta_{i \rightarrow j} \triangleq\left|M_{i \rightarrow j}\right|$, (14) can be expressed [4]

$$
\bar{M}_{j \rightarrow i}^{(l)}=\left(\prod_{i^{\prime} \in \overline{\mathcal{N}}(j) \backslash i} \alpha_{i^{\prime} \rightarrow j}^{(l)}\right) \Phi\left(\sum_{i^{\prime} \in \overline{\mathcal{N}}(j) \backslash i} \Phi\left(\beta_{i^{\prime} \rightarrow j}^{(l)}\right)\right)
$$

where the involutive function $\Phi(x) \triangleq-\ln \tanh (x / 2)$ can be precomputed and stored in LUT. Note however that an accurate LUT-based representation of $\Phi(x)$ may have important storage requirements.

From (21), a straightforward approximation considers that the VN message with minimum magnitude solely determines the resulting $\mathrm{CN}$ message. This approximation, denoted as min-sum (MS) approximation results in the $\mathrm{CN}$ messages

$$
\bar{M}_{j \rightarrow i}^{(l)} \approx\left(\prod_{i^{\prime} \in \overline{\mathcal{N}}(j) \backslash i} \alpha_{i^{\prime} \rightarrow j}^{(l)}\right) \min _{i^{\prime} \in \overline{\mathcal{N}}(j) \backslash i} \beta_{i^{\prime} \rightarrow j}^{(l)}
$$

However, expression (22) leads to overestimated check messages with respect to (21). Therefore, variants of the MS algorithm have been developed which consider a simple offset or scaling of the CN metric and are thus denoted as offset MS (OMS) or scaled MS (SMS), respectively. OMS and SMS CN messages are therefore

$$
\bar{M}_{j \rightarrow i}^{(l)} \approx\left(\prod_{i^{\prime} \in \overline{\mathcal{N}}(j) \backslash i} \alpha_{i^{\prime} \rightarrow j}^{(l)}\right) \max \left(\left(\min _{i^{\prime} \in \overline{\mathcal{N}}(j) \backslash i} \beta_{i^{\prime} \rightarrow j}^{(l)}\right)-\zeta, 0\right)
$$

and

$$
\bar{M}_{j \rightarrow i}^{(l)} \approx\left(\prod_{i^{\prime} \in \overline{\mathcal{N}}(j) \backslash i} \alpha_{i^{\prime} \rightarrow j}^{(l)}\right)\left(\min _{i^{\prime} \in \overline{\mathcal{N}}(j) \backslash i} \beta_{i^{\prime} \rightarrow j}^{(l)}\right) \zeta
$$

respectively where $\zeta$ denotes either the (positive) offset in (23) or the scaling factor $(0<\zeta<1)$ in $(24)$, respectively. 


\begin{tabular}{|c||c|c|c|}
\hline \multirow{2}{*}{ Configuration } & \multicolumn{2}{|c|}{ Demapping } & Decoding \\
\cline { 2 - 4 } & Iterative & Algorithm & Algorithm \\
\hline \hline 1 & No & Log MAP & Log SPA \\
\hline 2 & No & Log MAP & MS \\
\hline 3 & No & Log MAP & SMS $(\zeta=0.80)$ \\
\hline 4 & No & Max Log MAP & Log SPA \\
\hline 5 & Yes & Log MAP & Log SPA \\
\hline 6 & Yes & Log MAP & MS \\
\hline 7 & Yes & Log MAP & SMS $(\zeta=0.80)$ \\
\hline 8 & Yes & Max Log MAP & Log SPA \\
\hline \multicolumn{4}{|c}{ TABLE I } \\
\hline
\end{tabular}

EVALUATED LDPC-CODED CSK CONFIGURATIONS FOR ERROR RATE PERFORMANCE ANALYSIS

\section{Error rate performance analysis}

1) Parameters: Using previously presented algorithms, error rate performance has been evaluated in AWGN channel through simulations for various decoder configurations exposed in Table I. Rate $1 / 2$ binary LDPC codes with length $n=1200$ are built from optimized degree-distributions and progressive edge growth (PEG) matrix construction following [6]. Note that LDPC codes optimized for BICM-ID or BICM reception are considered in both respective cases. Block interleaving is used, 64-ary CSK signals are considered and signals are modelled directly at demodulator's output, assuming perfect orthogonality between CSK symbols. Also note that in every case, $I_{d e c}=50$, and in case of BICMID, demapping metrics are recomputed before each LDPC decoding iteration. For comparison purpose, we also present the performance results achieved by rate $1 / 2$ convolutionallycoded $^{3}$ and LDPC coded BPSK signals. The convolutional code is the CCSDS code (with octal generator polynomials coefficients $[133,171])$ and the LDPC code is that of GPS L1C subframe 2. The latter configuration corresponds to the GPS L1C (subframe 2) data component, while the former resemble Galileo data components.

2) Results: The error rate performance of configurations in Table I is depicted in Fig. 7. The importance of iterative demapping (with carefully optimized codes) is exposed. Under BICM-ID, max log MAP demapping is shown to lead to a degradation of $0.3 \mathrm{~dB}$ at $\mathrm{FER}=10^{-3}$. However, this degradation is less important with non-iterative demapping receivers, where the performance loss at $\mathrm{FER}=10^{-3}$ is only $0.1 \mathrm{~dB}$. On the other hand, suboptimal iterative LDPC decoding with the MS algorithm leads to a performance loss around $0.4 \mathrm{~dB}$ in both BICM-ID and BICM reception. However, SMS is able to recover most of the suboptimality and performs close to the SPA.

\footnotetext{
${ }^{3}$ For fair FER comparisons, the block length of the convolutionally-coded link is chosen equal to 1200 . Soft input Viterbi decoding is used and trellis termination is assumed.
}

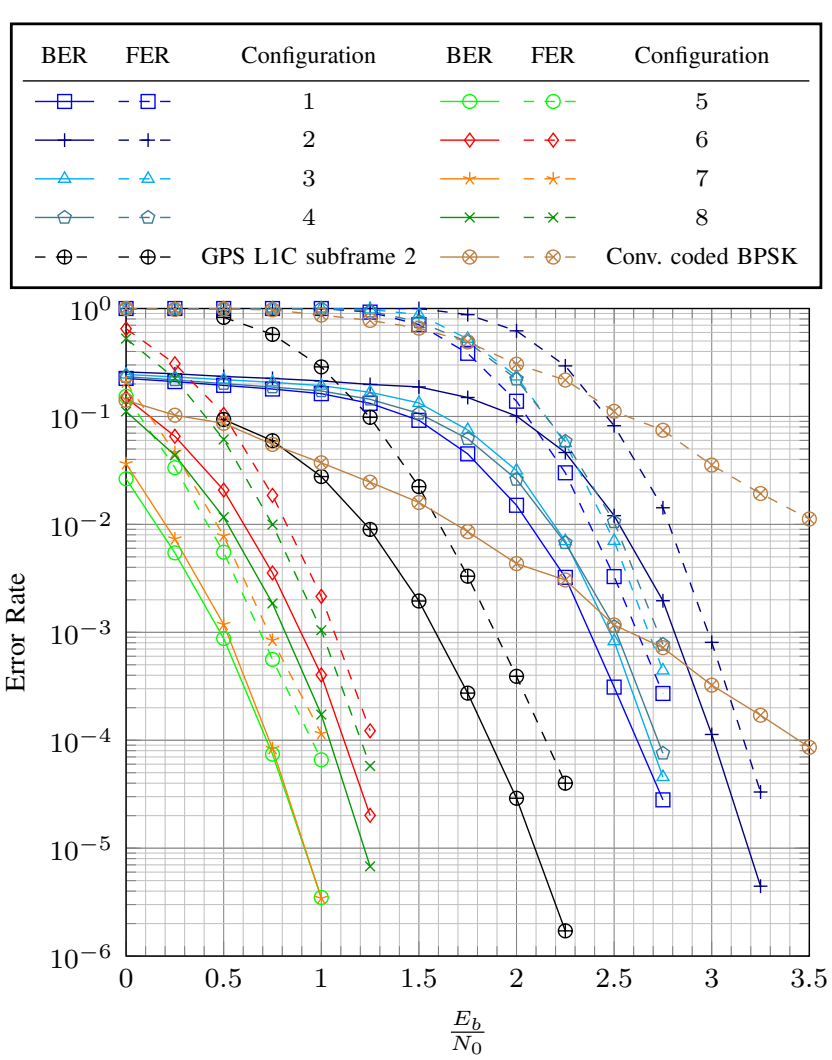

Fig. 7. Error rate performance of various coded CSK and BPSK links in AWGN channel

\section{RECEIVER COMPLEXITY ANALYSIS}

The computational complexity required to decode the transmitted GNSS data is considered here. We consider, as a measuring metric, the number of elementary mathematical operations needed to perform the tasks necessary for the decoding of received codewords. In the sequel, due to formatting constraints, we will sometimes denote $\mathbb{R} \pm$ and $\mathbb{R} \times$, the addition/subtraction and multiplication operations with operands in $\mathbb{R}$, respectively.

\section{A. Carrier wipe-off}

After the analog-to-digital conversion of the signal at intermediate frequency, a carrier wipe-off must be carried in order to convert the signal to baseband and compensate the residual Doppler shift. In coherent reception, the carrier wipeoff requires one real multiplication per sample. Therefore, $\alpha L$ real multiplications are required per modulated symbol.

\section{B. Coherent demodulation}

1) Processing at sample rate: Generally, the practical demodulation complexity depends on the ADC sampling rate. Whereas coherent DSSS/BPSK demodulation necessitates a single correlator, a $M$-branch correlator-bank is necessary for coherent $M$-ary CSK demodulation. Therefore, the complexity generally increases with the achievable data rate. Even if the demodulation of CSK signals can also be achieved in the Fourier domain, CSK demodulation is always more complex 
than DSSS/BPSK demodulation. The approximate number of operations per symbol required for coherent demodulation of DSSS/BPSK and CSK signals is shown in Table $\mathrm{II}^{4}$. The number of operations per bit can be straightforwardly obtained by dividing the values by the number of bits per symbol (1 for DSSS/BPSK, $U=\log _{2}(M)$ for CSK).

\begin{tabular}{|l||c|c|}
\hline & \# of mult. (in $\mathbb{R})$ & \# of add./sub. (in $\mathbb{R}$ ) \\
\hline \hline DSSS/BPSK & $\alpha L$ & $\alpha L-1$ \\
\hline CSK (correlators) & $\alpha M L$ & $M(\alpha L-1)$ \\
\hline CSK (FFT) & $4 \tilde{L}\left(\log _{2} \tilde{L}+1\right)$ & $2 \tilde{L}\left(3 \log _{2} \tilde{L}+1\right)$ \\
\hline CSK (RFFT) & $2 \tilde{L}\left[\log _{2}\left(\frac{\tilde{L}}{2}\right)+8\right]$ & $\tilde{L}\left[3 \log _{2}\left(\frac{\tilde{L}}{2}\right)+16\right]$ \\
\hline \multicolumn{2}{|c|}{ TABLE II }
\end{tabular}

NUMBER OF OPERATIONS PER SYMBOL REQUIRED FOR SAMPLE-RATE COHERENT DEMODULATION

In the case of (radix-2) FFT-based reception, we assumed that $\operatorname{FFT}\left(\mathbf{c}_{\mathrm{f}, \alpha}\right)^{*}$ is stored and need not be recomputed at every symbol. Therefore, only one FFT is needed. Note as well that Fourier-domain based demodulation requires complex operations and the corresponding real operations are obtained using the following conversion step; 1 complex multiplication requires 4 real multiplications and 2 real additions while 1 complex addition requires 2 real additions. Because in coherent reception, Fourier transforms can be applied to a real sequence, the computational complexity for the radix-2 FFT with complex-domain packing of real domain-coefficients [17] is also considered and denoted RFFT in Table II.

2) CSK demodulation processing at chip-rate: A rate reduction before CSK demodulation is of particular interest to limit the number of mathematical operations to be later performed. This can be achieved using a chip matched filter (CMF) prior the demodulation processing. Because of the rectangular pulse-shaping of chips in GNSS, the digital CMF reduces to a simple integrate-and-dump function which can be implemented with $(\alpha-1) L$ real additions per symbol. Assuming this prior CMF, and using $\tilde{L}^{\prime} \triangleq 2^{\left\lceil\log _{2} L\right\rceil}$, it is straightforward to show that the number of required operations for the (chip-rate) coherent demodulation of CSK signals is approximately reduced by $\alpha$, as depicted in Table III. For

\begin{tabular}{|l||c|c|}
\hline & \# of $\mathbb{R} \times$ & $\#$ of $\mathbb{R} \pm$ \\
\hline \hline DSSS/BPSK & $L$ & $L-1$ \\
\hline CSK (correlators) & $M L$ & $M(L-1)$ \\
\hline CSK (FFT) & $4 \tilde{L}^{\prime}\left(\log _{2} \tilde{L}^{\prime}+1\right)$ & $2 \tilde{L}^{\prime}\left(3 \log _{2} \tilde{L}^{\prime}+1\right)$ \\
\hline CSK (RFFT) & $2 \tilde{L}^{\prime}\left[\log _{2}\left(\frac{\tilde{L}^{\prime}}{2}\right)+8\right]$ & $\tilde{L}^{\prime}\left[3 \log _{2}\left(\frac{\tilde{L}^{\prime}}{2}\right)+16\right]$ \\
\hline \multicolumn{3}{|c}{ TABLE III }
\end{tabular}

NUMBER OF OPERATIONS PER SYMBOL REQUIRED FOR CHIP-RATE COHERENT DEMODULATION

$L=4092$ and $U=6$, the complexity of sample-rate and chip-rate demodulation (with digital CMF) processing are

\footnotetext{
${ }^{4}$ Note that, because PRN sequences have \pm 1 coefficients, multiplications can easily be avoided in correlator(-bank) based implementations (e.g. using exclusive-or of sign bits under sign/magnitude number representation).
}

compared as a function of $\alpha$ in Fig. 8. From Tables II and

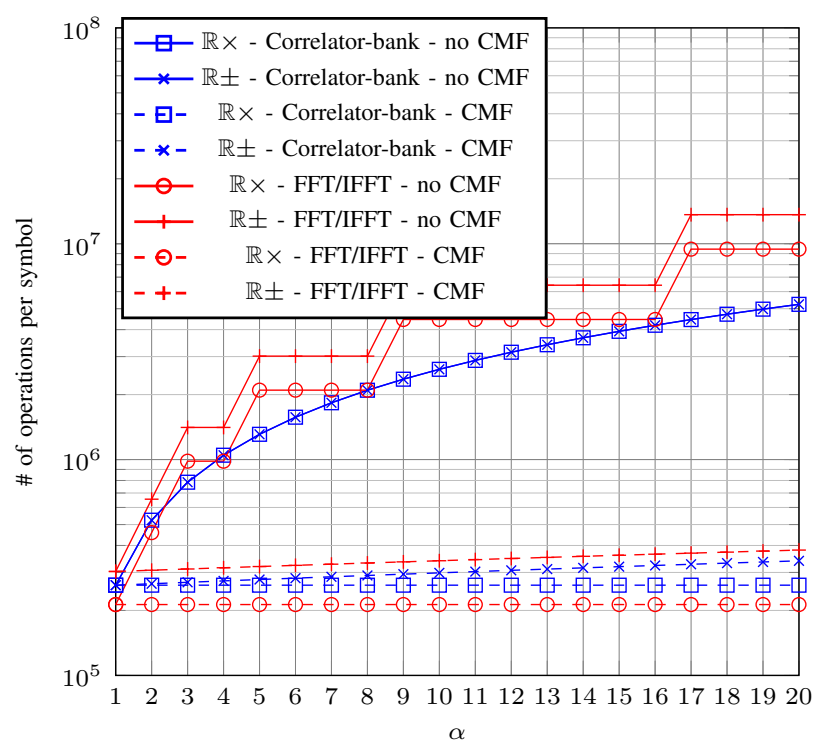

Fig. 8. Number of operations required for the chip-rate CSK demodulation with digital CMF and the sample-rate demodulation as a function of $\alpha(L=$ 4092, $U=6$ )

III, it can also be deduced that while correlator-bank based CSK demodulation is of typical interest for low-to-moderate CSK orders, Fourier-domain processing becomes mandatory for high CSK orders, as depicted in Fig. 9. This is because, unlike Fourier-domain demodulation, correlator-bank based demodulation complexity explicitly depends on $M$.

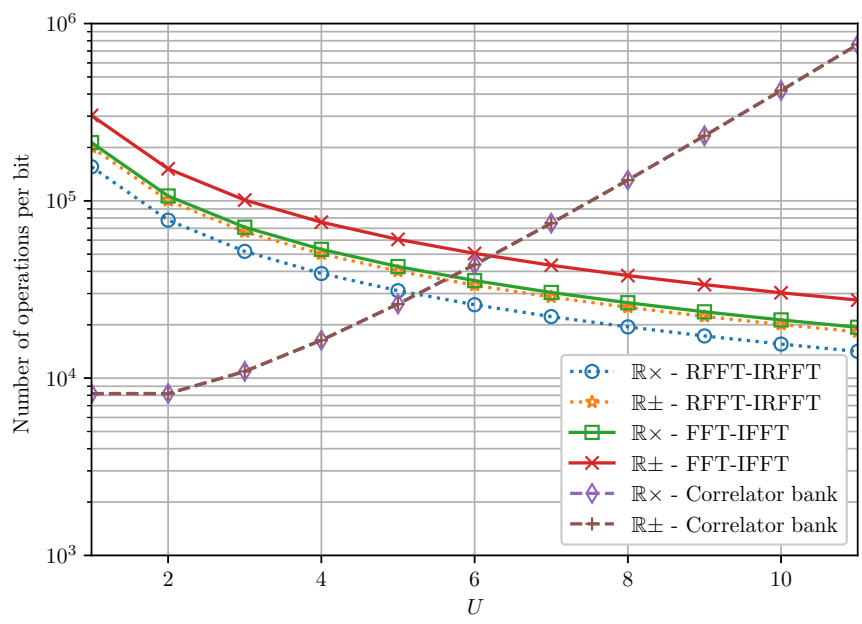

Fig. 9. Number of operations per bit required for chip-rate CSK demodulation $(L=4092)$

\section{Coherent CSK demapping}

Contrary to BPSK, for which demapping is straightforward since symbolwise and bitwise metrics are equivalent, CSK demapping can have significant complexity. Denoting $I_{d e m}$ the maximum number of demapper activations, the maximum 
number of operations required for iterative demapping is shown in Table IV. Note however that, as will be discussed in section V-D3, the practical number of activations can be lower than $I_{d e m}$. In BICM, the number of operations required

\begin{tabular}{|c|c|c|c|}
\hline Op. & Log MAP & $\begin{array}{l}\text { Log MAP using } \\
\max ^{\star}(.) \text { and LUT }\end{array}$ & Max Log MAP \\
\hline $\mathbb{R} \times$ & $M$ & $M$ & $M$ \\
\hline$>$ & & $I_{d e m} U(M-2)$ & $I_{d e m} U(M-2)$ \\
\hline $\exp ()$. & $I_{d e m} M$ & & \\
\hline $\mathbb{R} \pm$ & $\begin{array}{c}I_{\text {dem }} U(M-1) \\
+\left(I_{\text {dem }}-1\right) \times \\
{[U(M / 2+1)+1]}\end{array}$ & $\begin{array}{c}I_{\text {dem }} U(2 M-3) \\
\quad+\left(I_{\text {dem }}-1\right) \times \\
{[U(M / 2+1)+1]}\end{array}$ & $\begin{array}{c}I_{\text {dem }} U+ \\
\left(I_{\text {dem }}-1\right) \times \\
{[U(M / 2+1)+1]}\end{array}$ \\
\hline $\ln ()$. & $2 U I_{d e m}$ & & \\
\hline LUT & & $I_{d e m} U(M-2)$ & \\
\hline
\end{tabular}

TABLE IV

MAXIMUM NUMBER OF OPERATIONS REQUIRED FOR CSK BICM-ID DEMAPPING (PER SYMBOL)

for CSK demapping is expressed in Table V. From Table IV,

\begin{tabular}{|l||c|c|c|}
\hline Operation & Log MAP & $\begin{array}{c}\text { Log MAP using } \\
\max ^{\star}(.) \text { and LUT }\end{array}$ & Max log MAP \\
\hline \hline $\mathbb{R} \times$ & $M$ & $M$ & $U$ \\
\hline $\exp ()$. & $M$ & $U(2 M-3)$ & $U$ \\
\hline $\mathbb{R} \pm$ & $U(M-1)$ & & $U(M-2)$ \\
\hline $\ln ()$. & $2 U$ & $U(M-2)$ & \\
\hline$>$ & & $U(M-2)$ & \\
\hline LUT & \multicolumn{3}{|c}{ TABLE V }
\end{tabular}

NUMBER OF OPERATIONS REQUIRED FOR CSK BICM DEMAPPING (PER SYMBOL)

it can be observed that the complexity of iterative demapping is $\mathcal{O}\left(M \log _{2} M\right)$ per symbol and iteration. The complexity is dominated by real additions, except for the max log MAP approximation in non-iterative BICM demapping in which case comparisons dominates. Comparing Table V to Table III, for practical values of $(L, M)$, it can be inferred that the noniterative demapping complexity generally stands at least one order of magnitude below chip-rate demodulation complexity. Though still lower than chip-rate demodulation complexity, iterative demapping complexity can however become significant, as will be emphasized later. Also, in GNSS, while demodulation is typically performed with dedicated hardware, demapping and channel decoding may be executed using software resources. Therefore complexity reduction of iterative demapping still retains high interest and will be discussed in section VI.

\section{Binary LDPC decoding}

Binary error correction decoding has a complexity which is independent of the data modulation. For clarity, we consider separately the computational complexity of the steps used for iterative LDPC decoding; the VN processing, $\mathrm{CN}$ processing and codeword check.
1) $\mathrm{CN}$ processing: $\mathrm{CN}$ processing differs among presented algorithms. The classical log SPA originally requires computationally complex operations due to (14) but can be implemented without these at the cost of a single LUT using (21). On the other hand, the MS algorithm further decreases the computational complexity at the cost of a performance loss, by simplifying the $\mathrm{CN}$ message computation using (22). From (23) and (24), it can be observed that OMS and SMS add limited computational complexity increase with respect to MS. The required $\mathrm{CN}$ operations per codeword and per decoding iteration are depicted in Table VI where $\bar{d}_{c}$ is the average CN degree of the Tanner graph of $\mathbf{H}$.

\begin{tabular}{|l||c|c|c|c|}
\hline Operation & $\begin{array}{c}\text { log SPA } \\
\text { (with LUT) }\end{array}$ & MS & OMS & SMS \\
\hline \hline $\operatorname{sign}()$. & $m \bar{d}_{c}$ & $m \bar{d}_{c}$ & $m \bar{d}_{c}$ & $m \bar{d}_{c}$ \\
\hline$>$ & & $m\left(2 \bar{d}_{c}-3\right)$ & $m\left(2 \bar{d}_{c}-1\right)$ & $m\left(2 \bar{d}_{c}-3\right)$ \\
\hline$\oplus(1$ bit $)$ & $m\left(2 \bar{d}_{c}-1\right)$ & $m\left(2 \bar{d}_{c}-1\right)$ & $m\left(2 \bar{d}_{c}-1\right)$ & $m\left(2 \bar{d}_{c}-1\right)$ \\
\hline$||$. & $m \bar{d}_{c}$ & $m \bar{d}_{c}$ & $m \bar{d}_{c}$ & $m \bar{d}_{c}$ \\
\hline LUT & $2 m \bar{d}_{c}$ & & & \\
\hline $\mathbb{R} \pm$ & $m\left(2 \bar{d}_{c}-1\right)$ & & $2 m$ & \\
\hline $\mathbb{R} \times$ & & & & $2 m$ \\
\hline
\end{tabular}

TABLE VI

NUMBER OF OPERATIONS REQUIRED FOR CN PROCESSING PER CODEWORD AND ITERATION

2) VN processing: VN processing is the same in all algorithms considered here and required operations per codeword and decoding iteration are depicted in Table VII where $\bar{d}_{v}$ is the average $\mathrm{VN}$ degree of the Tanner graph of $\mathbf{H}$.

\begin{tabular}{|l||c|}
\hline Operation & For all algorithms \\
\hline \hline $\mathbb{R} \pm$ & $2 n \bar{d}_{v}$ \\
\hline \multicolumn{2}{|c||}{ TABLE VII }
\end{tabular}

NUMBER OF OPERATIONS REQUIRED FOR VN PROCESSING PER CODEWORD AND ITERATION

3) Codeword check: In order to limit the number of decoding iterations, an early stopping criteria is generally implemented which stops LDPC decoding as soon as the decoded sequence $\hat{\mathbf{x}}$ belong to the code. The number of operations required for each codeword check is negligible and will be omitted in the sequel. Therefore, the codeword check is a very attractive feature for complexity reduction since it strongly reduces the average number of decoding iterations $\bar{I}_{d e c}$ performed when the channel condition is sufficiently good. In BICM-ID, it can also reduce $\bar{I}_{d e m}$, the average number of demapper activations performed.

4) Overall LDPC decoding complexity: Combining Tables VI and VII, it can be observed that the overall complexity per codeword and iteration is linear in the LDPC matrix density, i.e. the number of non-zero coefficients $m \bar{d}_{c}=n \bar{d}_{v}$ of $\mathbf{H}$.

\section{E. Overall data recovery complexity}

1) Preamble: It is important to note that, thanks to the codeword check performed at every LDPC decoding iteration, 
the number of iterations performed differs, mainly based upon the channel quality. Therefore, in AWGN channel, a low $E_{b} / N_{0}$ is detrimental to the overall computational complexity for LDPC-coded links. This is intuitive because at low $E_{b} / N_{0}$, the decoding convergence to a valid codeword is slower or even unreachable. For this reason, it should be noted that BICM is not necessarily less computationally-intensive than BICM-ID, even though it is generally the case. Indeed, at low $E_{b} / N_{0}$, BICM-ID is able to converge faster than BICM reception, therefore, a significantly increased number of LDPC decoding iterations must be performed for the BICM receiver. This is depicted in Fig. 10.

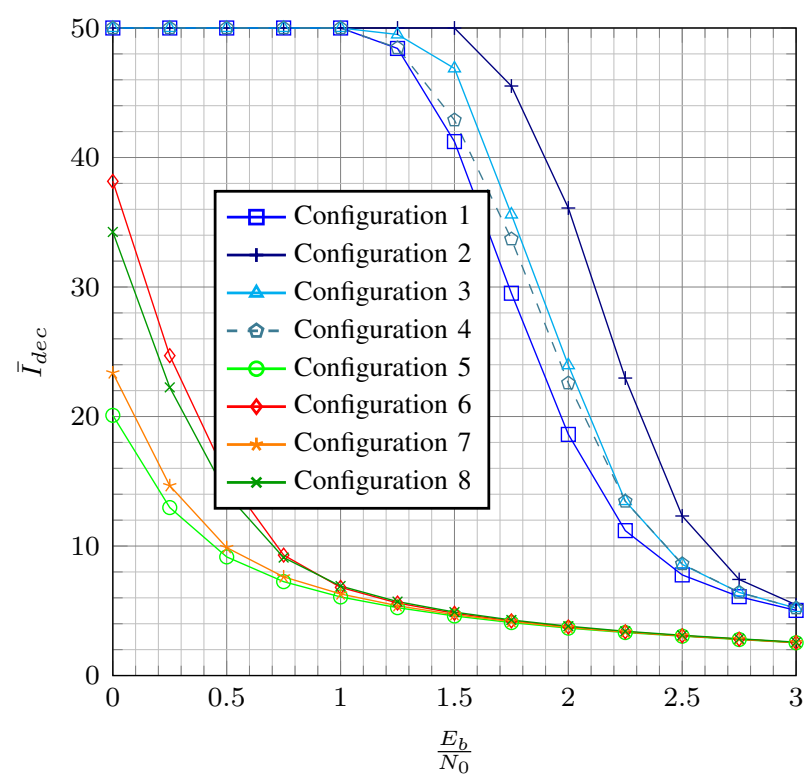

Fig. 10. Average number of LDPC decoding iterations $\bar{I}_{\text {dec }}$ performed for configurations of Table I in AWGN channel $\left(I_{d e c}=50\right)$

2) Scenario: The computational complexity for data recovery is a function of many variables, over which an exhaustive enquiry is not possible here for briefness. Here, we will assume $L=4092, \alpha=12$ and consider an AWGN channel with $E_{b} / N_{0}$ such that $\mathrm{FER}=10^{-1}$, depending on the receiver configuration. Such a channel condition is illustrative of a close-to-worst case reception scenario, for which the overall decoding complexity will be increased. Note however, that because of the $U$-fold data rate increase for CSK signals, keeping the same $E_{b} / N_{0}$ would necessitate an increase of the symbol energy requiring a raise of transmitted signal's power, in the scenario presented here. Several receiver configurations for 64-ary and 256-ary CSK signals have been evaluated. An LDPC-coded DSSS/BPSK link will also be considered for comparison. Configuration details are exposed in the first row of Table VIII, as well as in the sub-rows labelled "Info.".

3) Results: The equivalent number of operations per symbol (i.e per PRN sequence) necessary for data recovery is expressed in Table VIII. While enabling a $U$-fold data rate improvement over DSSS/BPSK, the use of the CSK modulation is shown to significantly increase the number of operations to be performed per PRN sequence duration. Especially, sample-rate demodulation exhibit the highest computational cost. While significantly reducing this cost, chiprate demodulation preceded by chip-matched filtering still necessitate an approximate five-fold increase of the number of operations required per symbol/PRN sequence, with respect to the DSSS/BPSK demodulation, in studied configurations. The dominance of demodulation complexity is concluded for CSK signals. Concerning DSSS/BPSK, complexity is dominated by both the carrier wipe-off and demodulation process. Also, from Table VIII, non-iterative max log MAP demapping is shown to have limited complexity, being of interest for lowend receivers. On the other hand, iterative log MAP demapping can have a high computational cost, especially when $M$ is high. Such a high $M$ is however typically of interest for a substantial data rate increase in GNSS.

\section{LOW-COMPLEXITY BICM-ID PROCESSING}

In Fig. 7, iterative demapping has been shown to bring substantial performance improvement. However, in Tables IV and VIII, the computational cost of iterative demapping was presented to be significant, especially compared to the cost of LDPC decoding. This is of special importance given the fact that, while demodulation/despreading is typically implemented in dedicated hardware, channel decoding (and potentially demapping) may be targeted for a software implementation in some GNSS receivers. Hence, hereafter, techniques for reducing iterative demapping computational cost with close to optimal error rate performance are exposed.

\section{A. Reduced-complexity demapping architecture}

It can be observed from Table IV, (12), (17) and (19) that, in BICM-ID, most of the computational complexity comes from the summations (and/or comparisons) processing the symbolwise metrics, and also from the combination of a priori LLR coming from the LDPC decoder. Those two steps have complexity $\mathcal{O}\left(M \log _{2} M\right)$ per symbol and iteration, assuming naive implementation. However, more effective implementations allowing reuse of intermediate common terms can be designed. This allows the computation of $\mathbf{b}_{\mu} \mathbf{L}_{\mathrm{a}}^{T}(\forall \mu)$ with only $M-U-1$ real additions. Also, the computations of symbol metric combinations can be implemented with only $3 M-2 U-4$ real additions for the log MAP processing. An example of circuit for the latter implementation is provided in Fig. 11, for $M=16$. Similar gains occur for $\max ^{\star}($.$) -based,$ or max log MAP implementations. Therefore, the reuse of intermediate common terms reduces the demapping complexity per symbol and iteration from $\mathcal{O}\left(M \log _{2} M\right)$ to $\mathcal{O}(M)$. The maximum number of real additions and comparisons becomes that exposed in Table IX. Note that these architectural changes do not affect demapping performance.

\section{B. Reduced-complexity demapping schedule}

Because iterative demapping complexity is linear in $\bar{I}_{d e m}$, a simple strategy for complexity reduction aims at limiting the maximum number of demapping activations. While the 


\begin{tabular}{|c|c|c|c|c|c|c|c|}
\hline \multirow{2}{*}{\multicolumn{2}{|c|}{ Configurations $\rightarrow$}} & \multirow{2}{*}{ LDPC/BPSK } & \multicolumn{3}{|c|}{ BICM-ID LDPC-CSK } & \multicolumn{2}{|c|}{ BICM LDPC-CSK } \\
\hline & & & 64-CSK & 64-CSK & 256-CSK & 64-CSK & 256-CSK \\
\hline $\begin{array}{l}\text { Carrier } \\
\text { wipe-off }\end{array}$ & $\mathbb{R} \times$ & 49104 & 49104 & 49104 & 49104 & 49104 & 49104 \\
\hline $\begin{array}{l}\text { Chip-matched } \\
\text { filtering }\end{array}$ & $\mathbb{R} \pm$ & & & 45012 & 45012 & 45012 & 45012 \\
\hline \multirow[t]{3}{*}{ Demodulation } & Info. & Sample-rate & $\begin{array}{l}\text { Sample-rate, } \\
\text { correlator-bank }\end{array}$ & $\begin{array}{c}\text { Chip-rate, } \\
\text { correlator-bank }\end{array}$ & $\begin{array}{c}\text { Chip-rate, } \\
\text { Fourier domain } \\
(R F F T)\end{array}$ & $\begin{array}{c}\text { Chip-rate, } \\
\text { correlator-bank }\end{array}$ & $\begin{array}{c}\text { Chip-rate, } \\
\text { Fourier domain } \\
(R F F T)\end{array}$ \\
\hline & $\mathbb{R} \times$ & 49104 & 3142656 & 261888 & 155648 & 261888 & 155648 \\
\hline & $\mathbb{R} \pm$ & 49103 & 3142592 & 261824 & 200704 & 261824 & 200704 \\
\hline \multirow{4}{*}{ Demapping } & Info. & $\log M A P$ & \multicolumn{3}{|c|}{ Iterative $\log M A P$ using $\max ^{\star}($.$) and L U T$} & \multicolumn{2}{|c|}{ Non-iterative max $\log M A P$} \\
\hline & $\mathbb{R} \times$ & 1 & 64 & 64 & 256 & 6 & 8 \\
\hline & $\mathbb{R} \pm$ & & 16883 & 16883 & 90857 & 6 & 8 \\
\hline & $>$ & & 6696 & 6696 & 36576 & 372 & 2032 \\
\hline \multirow{4}{*}{$\begin{array}{l}\text { Channel } \\
\text { decoding }\end{array}$} & Info. & $\begin{array}{l}\text { Log SPA with LUT, GPS } \\
\text { L1C subframe } 2 \text { code }\end{array}$ & \multicolumn{3}{|c|}{ Log SPA with LUT, BICM-ID optimized code } & \multicolumn{2}{|c|}{ SMS, BICM optimized code } \\
\hline & $\mathbb{R} \times$ & & & & & 108 & 144 \\
\hline & $\mathbb{R} \pm$ & 310 & 810 & 810 & 1080 & 862 & 1149 \\
\hline & $>$ & & & & & 700 & 933 \\
\hline
\end{tabular}

NUMBER OF MAIN OPERATIONS PER SYMBOL (I.E. PER PRN SEQUENCE) REQUIRED FOR DATA RECOVERY

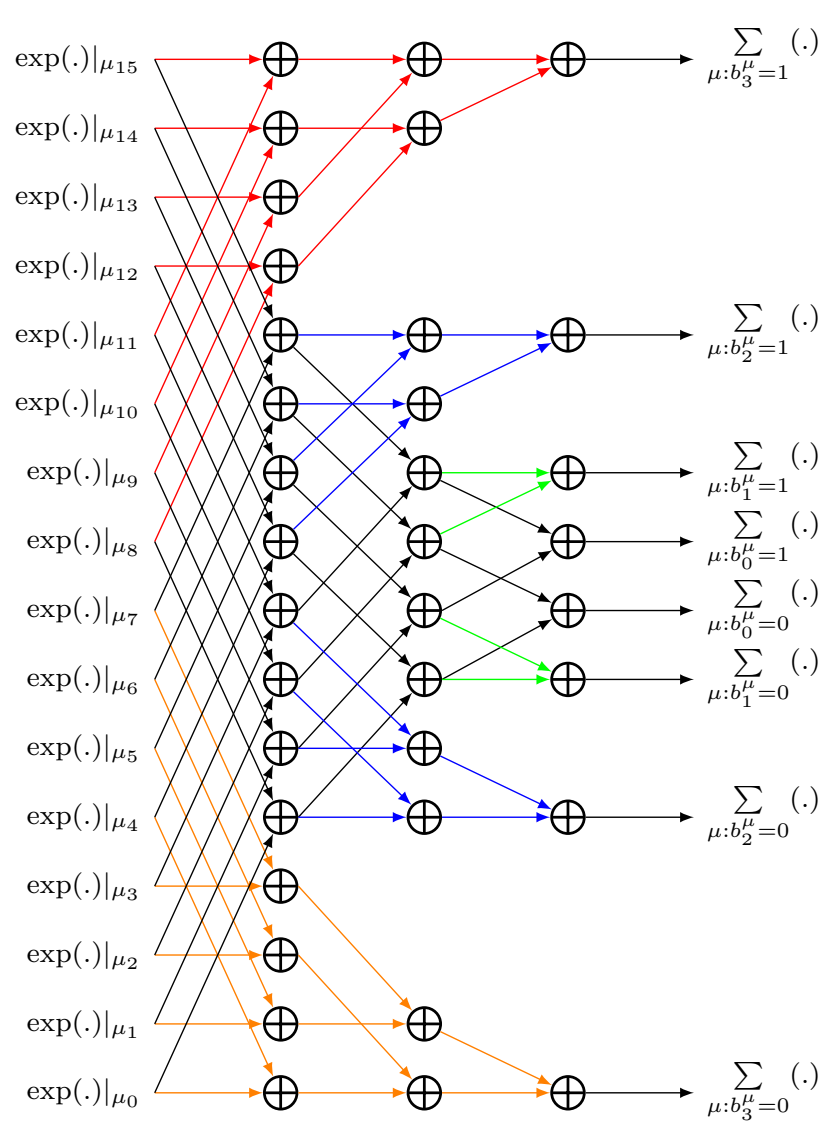

Fig. 11. Partial-sum based combination of symbolwise metrics for demapping $(M=16)$

\begin{tabular}{|c|c|c|c|}
\hline & Log MAP & $\begin{array}{c}\text { Log MAP using } \\
\max ^{\star}(.) \text { and } \\
\text { LUT }\end{array}$ & Max log MAP \\
\hline \multirow{2}{*}{$\operatorname{BICM} \frac{>}{\mathbb{R} \pm}$} & & $3 M-2 U-4$ & $3 M-2 U-4$ \\
\hline & $3 M-U-4$ & $6 M-3 U-8$ & \\
\hline \multirow[t]{2}{*}{$\begin{array}{l}\text { BICM- > } \\
\text { ID }\end{array}$} & & $\begin{array}{c}I_{d e m}(3 M- \\
2 U-4)\end{array}$ & $\begin{array}{c}I_{d e m}(3 M- \\
2 U-4)\end{array}$ \\
\hline & $\begin{array}{c}I_{\text {dem }} \times \\
(3 M-U-4) \\
+\left(I_{\text {dem }}-1\right) \times \\
{[2 M-1]}\end{array}$ & $\begin{array}{c}I_{\text {dem }} \times \\
(6 M-3 U-8) \\
+\left(I_{\text {dem }}-1\right) \times \\
{[2 M-1]}\end{array}$ & $\begin{array}{c}I_{\text {dem }} U+ \\
\left(I_{\text {dem }}-1\right) \times \\
{[2 M-1]}\end{array}$ \\
\hline
\end{tabular}

\section{TABLE IX}

MAXIMUM NUMBER OF MAIN OPERATIONS NEEDED FOR DEMAPPING REUSING INTERMEDIATE COMMON TERMS

optimal BICM-ID activates the demapper before every new LDPC decoding iteration, low-complexity iterative demapping discussed here activates the demapper according to a specific schedule (cf. Fig. 12).

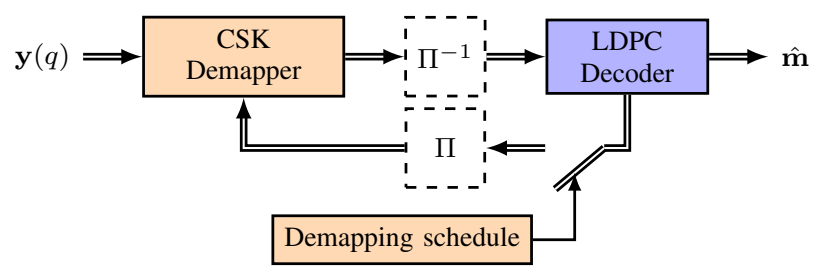

Fig. 12. Structure of BICM-ID decoder with incomplete demapping activation schedule 


\section{Optimized trivial demapping schedules}

Assuming incompleteness of the iterative demapping activation schedule, i.e. $I_{d e m}<I_{d e c},\left(\begin{array}{c}I_{d e c}-1 \\ I_{d e m}-1\end{array}\right)$ schedules are possible. Therefore, the prediction of the optimal schedule with respect to error rate performance generally necessitates huge simulation ressources under exhaustive search. In [18], a regular activation spacing (cf. Fig. 13) was advised for LDPC codes with optimized irregular degree distributions when $n \rightarrow \infty$. In the case of limited codeword length, we observed that the iteration spacing between demapper activations should be carefully chosen. In general, scheduling should also vary depending on the channel quality (e.g. $E_{b} / N_{0}$ in AWGN channel).

Demapping activation

Decoding iteration

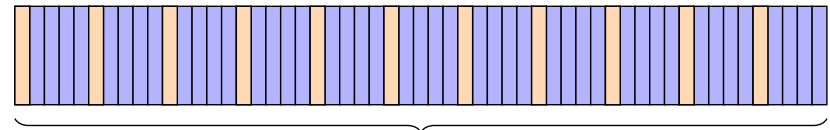

$$
I_{d e c}+I_{d e m}
$$

Fig. 13. Illustration of an incomplete regularly spaced iterative demapping schedule with maximal activation spacing

Therefore, optimal regularly spaced demapping schedules are here proposed. Under a constrained maximum number of demapping activations, limiting to regularly spaced activation schedules allows to drastically reduce the number of candidate schedules. An optimal schedule for a given error rate performance criterion can therefore be selected through exhaustive search in this reduced set of schedules.

For $M=64$, reference configurations and $I_{\text {dem }} \in$ $\{2,3,5,10\}$, regularly-spaced schedules minimizing the $E_{b} / N_{0}$ for FER $\approx 10^{-3}$ have performance depicted in Fig. 14. Note that an early error floor can occur because of a high activation spacing. This behaviour can be reduced by using an adaptive activation spacing depending upon $E_{b} / N_{0}$. While it can be seen that performance degrades with decreased $I_{\text {dem }}$, we can observe from Fig. 15 a diminishing return of the reduction of the number of practical demapping activations, which determines the relative demapping complexity gain. Indeed, as a consequence of the codeword check performed at every iteration, the demapping activation budget is rarely consumed for sufficiently high values of $E_{b} / N_{0}$.

For optimized schedules, the number of main operations per symbol for log MAP demapping using $\max ^{\star}($.$) and LUT-$ based implementation at FER $=10^{-1}$ is depicted in Table X. The reuse of common terms exposed in section VI-A provides a two-fold reduction of the number of operations needed for demapping. Additionally, an approximate three-fold decrease of the latter is observed by limiting $I_{d e m}$ to 10 instead of 50. A further decrease of $I_{d e m}$, while bringing additional complexity reduction would increasingly impact the error rate performance as illustrated in Fig. 14.

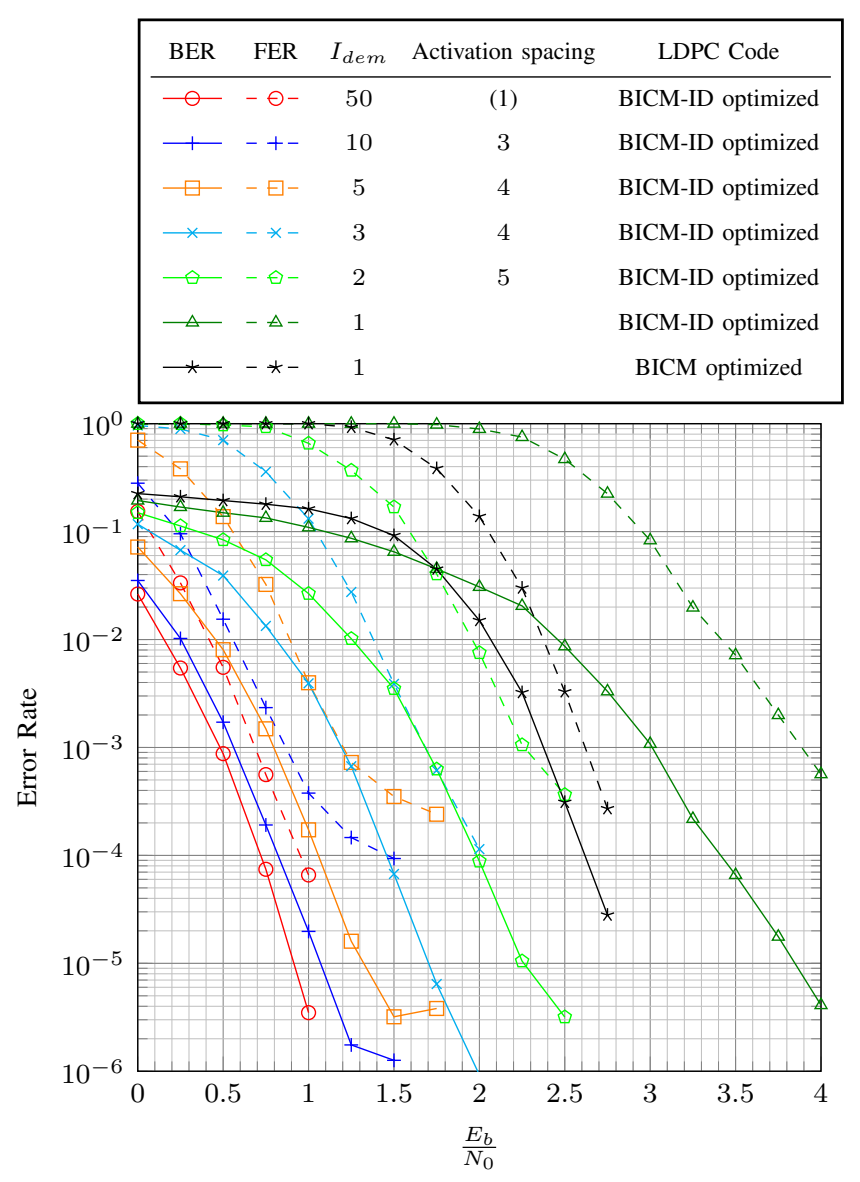

Fig. 14. Error rate performance in AWGN channel for BICM-ID receivers using regularly-spaced schedules optimized for $\mathrm{FER} \approx 10^{-3}$

\begin{tabular}{|c|c|c|c|c|c|c|c|}
\hline \multicolumn{2}{|c|}{$I_{\text {dem }} \rightarrow$} & 50 & 10 & 5 & 3 & 2 & 1 \\
\hline \multirow{2}{*}{$\begin{array}{l}\text { Naive } \\
\text { impl. }\end{array}$} & $\mathbb{R}+$ & 16883 & 5970 & 3977 & 2648 & 1699 & 750 \\
\hline & $>$ & 6696 & 2418 & 1637 & 1116 & 744 & 372 \\
\hline \multirow{2}{*}{$\begin{array}{l}\text { Reuse of } \\
\text { common } \\
\text { terms }\end{array}$} & $\mathbb{R}+$ & 8603 & 3025 & 2007 & 1328 & 843 & 358 \\
\hline & $>$ & 3168 & 1144 & 774 & 528 & 352 & 176 \\
\hline
\end{tabular}

NUMBER OF MAIN OPERATIONS REQUIRED PER SYMBOL/PRN SEQUENCE FOR DEMAPPING USING A LOG SPA ALGORITHM WITH $\max ^{\star}($.) AND LUT

\section{CONCLUSION}

Binary LDPC-coded CSK signals constitute an attractive proposal for a significant increase of data rate in GNSS. In this article, we illustrated the computational complexity needed at receiver side for their data recovery. CSK demodulation processing at chip rate is shown able to significantly reduce the computational cost of coded CSK data recovery. In spite of this, an approximate five-fold increase of the total number of operations per symbol/PRN sequence was observed for studied cases, with respect to the classical coded DSSS/BPSK data recovery requirements. This is to be put in perspective with the $U$-fold data rate improvement coming without altering the overall signals' spectrum and orthogonality properties. Various 


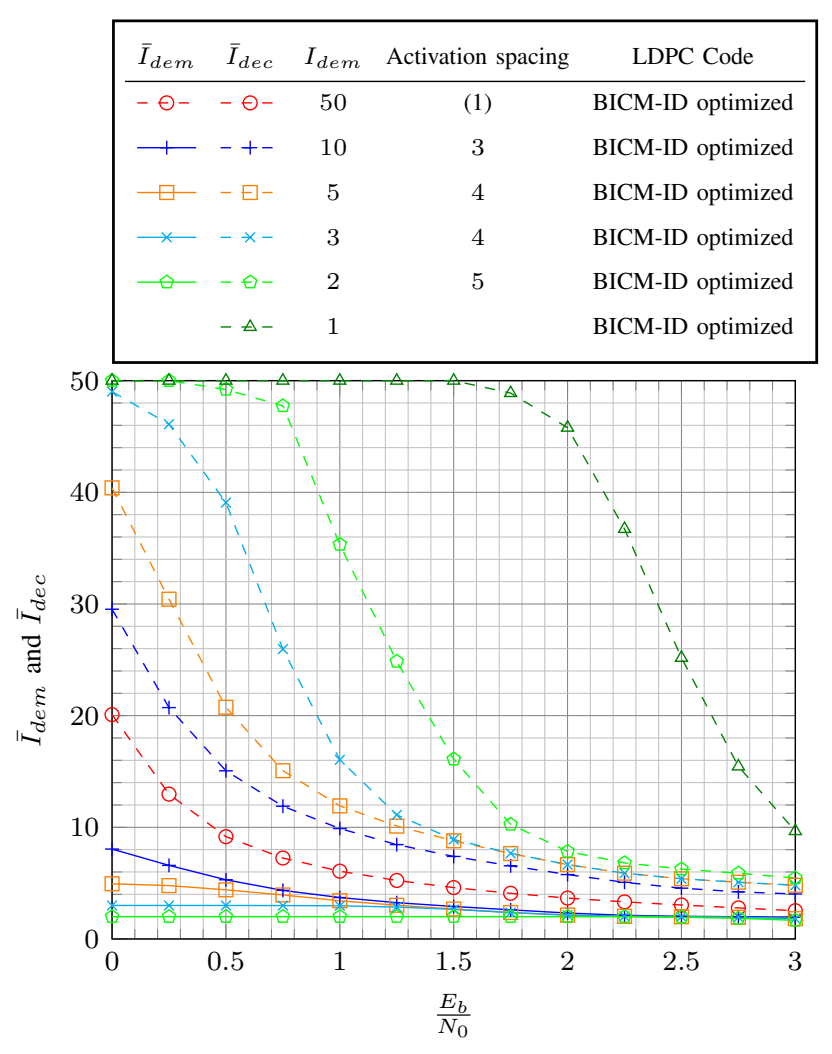

Fig. 15. Average number of LDPC decoding iterations (dashed lines) and demapping activations (solid lines) performed for regularly-spaced schedules optimized for $\mathrm{FER} \approx 10^{-3}\left(I_{d e c}=50\right)$

coded CSK implementation decoding strategies have been discussed with their performance and complexity. Finally, lowcomplexity iterative demapping has been proposed for LDPCcoded CSK signals. For the scenario exposed, a $0.2 \mathrm{~dB}$ loss of coding gain at $\mathrm{FER}=10^{-3}$ was traded for an approximate six-fold reduction of the number of operations required at a close-to worst case $\mathrm{FER}=10^{-1}$.

\section{REFERENCES}

[1] G. M. Dillard, M. Reuter, J. Zeidler, and B. Zeidler, "Cyclic code shift keying: A low probability of intercept communication technique," IEEE Transactions on Aerospace and Electronic Systems, vol. 39, no. 3, pp. 786-798, Jul. 2003.

[2] Japan Aerospace Exploration Agency, Quasi-Zenith Satellite System Interface Specification - Centimeter Level Augmentation Service. Nov. 5, 2018.

[3] T. Richardson and R. Urbanke, Modern Coding Theory. New York, USA: Cambridge University Press, 2008.

[4] W. Ryan and S. Lin, Channel Codes: Classical and Modern. Cambridge University Press, 2009.

[5] A. J. Garcia-Peña, D. Salós, O. Julien, L. Ries, and T. Grelier, "Analysis of the use of CSK for future GNSS signals," in Proceedings of ION GNSS+, Sep. 2013, pp 1461-1479.
[6] R. Chauvat, A. Garcia Pena, M. Anghileri, J. Floch, and M. Paonni, "Ultra-sparse binary LDPC codes with CSK signals for increased data rates in future GNSS,' in Proceedings of ESA NAVITEC, Dec. 2018, pp. 1-11.

[7] K. Nakakuki and R. Hirokawa, "An efficient acquisition method for the CSK signal of QZSS LEX," in Proceedings of ION GNSS+, Nashville, TN, Sep. 2013, pp. 340-347.

[8] R. Link and V. C. M. Leung, "Design, analysis, and DSP-based implementation of a code-phase-shift keying receiver for wireless local area network applications," International Journal of Wireless Information Networks, vol. 5, no. 3, pp. 187-202, Jul. 1, 1998.

[9] A. V. Veitsel, L. V. Purto, A. S. Lebedinsky, and D. A. Rubtsov, "Method and system for demodulating and tracking of csk-modulated signals," pat. 20180239026A1, Aug. 23, 2018.

[10] S. Che, P. Wang, L. Ping, and X. Wang, "Lowcomplexity soft-input soft-output demodulation of orthogonal modulation," IEEE Communications Letters, vol. 11, no. 12, pp. 919-921, Dec. 2007.

[11] S. Che and S. Tong, "Low-complexity LDPC coded BICM-ID with orthogonal modulations," Electronics Letters, vol. 45, no. 16, pp. 845-846, Jul. 2009.

[12] R. Tanner, "A recursive approach to low complexity codes," IEEE Transactions on Information Theory, vol. 27, no. 5, pp. 533-547, Sep. 1981.

[13] N. C. Shivaramaiah and A. G. Dempster, "On the baseband hardware complexity of modernized GNSS receivers," in Proceedings of IEEE ISCAS, May 2010, pp. 3565-3568.

[14] J. Nurmi, E. S. Lohan, S. Sand, and H. Hurskainen, Eds., GALILEO Positioning Technology, Signals and Communication Technology, Springer Netherlands, 2015.

[15] D. M. Akos and M. Pini, "Effect of sampling frequency on GNSS receiver performance," Navigation, vol. 53, no. 2, pp. 85-95, 2006.

[16] G. Caire, G. Taricco, and E. Biglieri, "Bit-interleaved coded modulation," IEEE Transactions on Information Theory, vol. 44, no. 3, pp. 927-946, May 1998.

[17] J. G. Proakis and D. G. Manolakis, Digital Signal Processing. Pearson Prentice Hall, 2007.

[18] L. Schmalen, S. ten Brink, and A. Leven, "Analysis and optimization of iteration schedules for LDPC coded modulation and detection," in Proceedings of IEEE ISTC, Aug. 2012, pp. 210-214. 